\title{
Volume and Characteristics of Intracerebral Hemorrhage with Direct Oral Anticoagulants in Comparison with Warfarin
}

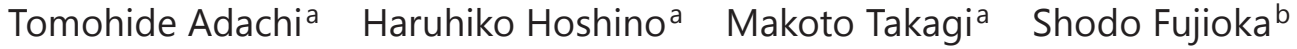 \\ Saiseikai Stroke Research Group \\ a Department of Neurology, Tokyo Saiseikai Central Hospital, Tokyo, Japan; ${ }^{b}$ Department of \\ Neurosurgery, Saiseikai Misumi Hospital, Uki-city, Japan
}

\author{
Keywords \\ Hemorrhage associated with oral anticoagulation · Anticoagulants · Intracerebral \\ hemorrhage
}

\begin{abstract}
Background: Patients undergoing anticoagulation therapy often experience intracerebral hemorrhages (ICHs), and warfarin in particular is known to increase hematoma expansion in ICHs, which results in a poor outcome. Recent studies reported that, in comparison with warfarin, direct oral anticoagulants (DOACs) cause fewer ICHs with better functional outcome. However, since it is still unknown whether DOACs are associated with a smaller hematoma volume of ICHs, we aimed to compare the volume, hematoma expansion, and outcomes associated with ICHs treated with DOACs and warfarin. Methods: We performed a prospective multicenter cross-sectional study. The subjects included patients with acute ICHs who received either DOACs or warfarin. We evaluated the clinical characteristics, and measured initial and follow-up ICH volumes. The volume of ICHs and hematoma expansion were compared between the DOAC and warfarin groups. Mortality and modified Rankin score at discharge were evaluated as outcomes. Results: There were 18 patients in the DOAC group and 71 in the warfarin group. The baseline characteristics were similar between the 2 groups. Initial median hematoma volume of ICHs in the DOAC group was significantly lower than that in the warfarin group (6.2 vs. $24.2 \mathrm{~mL}$, respectively; $p=0.04$ ). In cases involving follow-up computed tomography scanning, the median hematoma volume of ICHs at follow-up was lower in the DOAC group than in the warfarin group (initial: DOACs 4.4 vs. warfarin $13.5 \mathrm{~mL}$; follow-up: 5.0 vs. $18.4 \mathrm{~mL}$, respectively; $p=0.05$ ). Further, the hematoma in ICHs associated with DOACs did not expand. Although the mortality of ICHs associated with DOACs (11\%) was lower than that
\end{abstract}


associated with warfarin (24\%), this difference was not statistically significant. The univariate analysis showed that the anticoagulant type (DOACs vs. warfarin) and sex (male vs. female) were associated with $\mathrm{ICH}$ volume. The multivariable linear regression showed that the use of DOACs (compared to warfarin; $\beta$ : $-0.23, p=0.03$ ) and female sex (compared to male; $\beta$ : -0.25 , $p=0.02$ ) were associated with a small hematoma volume. Conclusions: Based on the results of the present study, in terms of the risks associated with ICHs, the use of DOACs appears to be safer than warfarin for anticoagulation therapy. Further studies are required to validate these findings.

(C) 2017 The Author(s)

Published by S. Karger AG, Basel

\section{Introduction}

Patients undergoing anticoagulation therapy often experience intracerebral hemorrhages (ICHs). Further, anticoagulation therapy with warfarin, in particular, increases hematoma expansion in ICHs, which leads to a poor outcome [1-4]. The incidence of ICHs is higher in warfarin-treated Asian populations in comparison with non-Asian populations [5, 6]. Recently, it has become possible to use direct oral anticoagulants (DOACs) as anticoagulation therapy for atrial fibrillation. All clinical trials using DOACs reported a significantly lower incidence of ICHs [7-10], which was evident in Asian populations [11-13]. In several small Japanese studies $[14,15]$, DOACs were associated with fewer ICHs and better functional outcomes compared with warfarin. However, the hematoma volume of ICHs and hematoma expansion were not statistically compared between those treated with DOACs and those treated with warfarin. Therefore, it has not been established if DOACs are associated with smaller hematoma volume in ICHs or if the associated functional outcomes are better with DOACs than warfarin. Thus, the aim of the current study was to clarify and compare the characteristics of ICHs associated with DOACs and warfarin, by prospectively observing hematoma volume, hematoma expansion, and clinical outcomes of ICHs.

\section{Methods}

\section{Subjects}

The Saiseikai Stroke Database is a registry of data of acute stroke patients collected during the first 7 days from onset. The Saiseikai Stroke Research Group consists of 25 hospitals belonging to the Social Welfare Organization Saiseikai Imperial Gift Foundation in Japan. Our group was the first to register with the Saiseikai Stroke Database on April 1, 2013. We collected patient data and stroke profiles, including age, sex, and stroke type (ischemic stroke, hemorrhagic stroke, or subarachnoid hemorrhage). Hemorrhagic strokes were classified as hypertensive, amyloid angiopathy, and other factors. Subarachnoid hemorrhages were classified as aneurysmal and other factors. During the first year, while we recorded stroke type alone, we subsequently began recording all baseline clinical characteristics, treatments, and prognoses at discharge from the second year onward. Therefore, in the current study, we selected consecutive patients with ICHs entered into the database from April 1, 2014 to March 31, 2015. This study was approved by the medical ethics board in each institute of the Saiseikai Stroke Research Group. Since we obtained general consent to use clinical data from each subject on admission, individual written informed consent was not obtained at the time of the study based on the Ethical Guideline for Medical and Health Research Involving Human Subjects outlined by the Ministry of Education, Culture, Sports, Science and Technology and the Ministry of Health, Labor and Welfare in Japan. 


\section{Risk Factors and Clinical Assessments}

We recorded data pertaining to hypertension, diabetes mellitus, dyslipidemia, chronic kidney disease, congestive heart failure, smoking status, and alcohol intake. The diagnosis of vascular risk factors (hypertension, diabetes mellitus, and dyslipidemia) was made according to the Japanese diagnostic criteria. Hypertension was defined as a history of hypertension or use of medication for hypertension, as were diabetes and dyslipidemia. Chronic kidney disease was defined as an estimated glomerular filtration rate $<60 \mathrm{~mL} / \mathrm{min}$; proteinuria was not assessed. Congestive heart failure was defined as a history of heart failure and its current treatment. Smoking was defined as current smoking status and alcohol intake was defined as the consumption of more than 1 drink per day.

We recorded the current medications of each patient on admission. Antithrombotic agents included the following: warfarin, DOACs (dabigatran, apixaban, rivaroxaban, and edoxaban), aspirin, clopidogrel, cilostazol, antihypertensive agents, statins, and oral hypoglycemic agents.

On admission, we recorded consciousness disturbance using the Japan Coma Scale [16], as well as systolic and diastolic blood pressure, and calculated the onset time of stroke and arrival time at the hospital, i.e., the onset-to-arrival time. We recorded all treatments performed for stroke. In cases of hemorrhagic stroke, the use of either operative therapy or conservative therapy was recorded. At discharge, the modified Rankin Scale (mRS) score and death due to any cause were recorded as prognostic outcomes. Further, we also recorded medication at discharge, namely treatment with antiplatelet agents, warfarin, DOACs, antihypertensive agents, and statins.

\section{Measurement of Hematoma Volume}

We obtained brain computed tomography (CT) images from all patients with ICHs on admission, and follow-up brain CT images from all patients who were available for assessment. We calculated the volume of hematoma using the ABC/2 method from axial CT images [17]. The measurement of hematoma, which was an initial assessment, was not performed in a blinded fashion.

\section{Statistical Analysis}

Baseline characteristics were compared between the DOAC and warfarin groups using the $\chi^{2}$ test and Student $t$ test. We compared the actual measured hematoma volume of the initial CT images between the DOAC group and the warfarin group using the Mann-Whitney $\mathrm{U}$ test. The frequency of hematoma volume expansion between the initial and follow-up CT scans was compared between the DOAC and warfarin groups using the $\chi^{2}$ test, while changes in hematoma volume between initial and follow-up CT scans were assessed using the Wilcoxon signed-rank test. Hematoma volume was converted by logarithmic transformation to satisfy statistical normality. Univariate and multivariable linear regression analyses were performed using logarithmic-transformed hematoma volume and anticoagulants as the dependent and independent variables, respectively. Other independent variables were stroke characteristics associated with ICH volume, risk factors of cerebral hemorrhage, and factors related to anticoagulant therapy. We selected age, sex, and histories of hypertension and cerebral infarction. The mRS score and the frequency of death at discharge were compared between the DOAC and warfarin groups using the $\chi^{2}$ test. All statistical analyses were performed using JMP 10 software (SAS Institute, Co. Ltd., Cary, NC, USA). 
Table 1. Baseline characteristics of DOAC-ICHs and warfarin-ICHs

\begin{tabular}{lccc}
\hline Variables & DOAC $(n=18)$ & Warfarin $(n=71)$ & $p$ value \\
\hline Age, years & $76.3 \pm 2.3$ & $77.9 \pm 1.2$ & 0.55 \\
Male & $10(56)$ & $40(56)$ & 0.95 \\
Onset-admission time, h & $1.8(1-3.4)$ & $1.7(1-5.4)$ & 0.82 \\
Level of consciousness (JCS) & $13(72)$ & $38(54)$ & 0.28 \\
Systolic blood pressure, mm Hg & $170 \pm 27$ & $164 \pm 29$ & 0.44 \\
Diastolic blood pressure, mm Hg & $96 \pm 21$ & $90 \pm 22$ & 0.26 \\
Hypertension & $17(94)$ & $56(79)$ & 0.18 \\
Diabetes & $3(17)$ & $9(13)$ & 0.70 \\
Dyslipidemia & $6(33)$ & $15(21)$ & 0.35 \\
Smoking (never) & $12(67)$ & $55(77)$ & 0.63 \\
Atrial fibrillation & $13(72)$ & $41(58)$ & 0.43 \\
Congestive heart failure & $1(6)$ & $20(28)$ & 0.06 \\
CKD/HD & $2(11)$ & $12(17)$ & 0.72 \\
Past stroke & & & - \\
$\quad$ Cerebral infarction & $5(28)$ & $21(30)$ & - \\
$\quad$ Cerebral hemorrhage & $2(11)$ & $5(7)$ & - \\
$\quad$ Both & 0 & $1(1)$ & 0.16 \\
Hypertensive hemorrhage & $16(88)$ & $52(73)$ & - \\
$\quad$ Amyloid angiopathy & 0 & $3(4)$ & - \\
Hemorrhage region & $11(61)$ & $29(41)$ & - \\
$\quad$ Deep & 0 & $19(27)$ & 0.04 \\
Subcortical & $5(28)$ & $13(18)$ & 0.57 \\
Infratentorial & $23(14-34)$ & $16(9-28)$ & \\
Hospital stay, days & $26(21-37)$ & $21(10-36)$ & \\
Hospital stay (alive outcome), days & $2(2-2)$ & & $-14)$ \\
Hospital stay (fatal outcome), days & & & \\
\hline
\end{tabular}

Figures are means $\pm \mathrm{SD}$, medians with IQRs in parentheses or numbers with percentages in parentheses. IQR, interquartile range; JCS, Japan Coma Scale [16]; CKD/HD, chronic kidney disease/hemodialysis; DOAC, direct oral anticoagulant; ICH, intracranial hemorrhage.

\section{Results}

\section{Baseline Characteristics}

A total of 10,524 patients were included in the registry, of whom 1,193 experienced ICH. The mean age of the patient cohort in the current study was $71.2 \pm 13.9$ years, and included 682 men (mean age: $68.4 \pm 13.4$ years) and 511 women (mean age: $75.1 \pm 13.3$ years). Hypertensive hemorrhage occurred in 802 cases (76.9\%), while amyloid angiopathy occurred in 58 cases $(5.6 \%)$.

On admission, 89 patients were prescribed oral anticoagulants, namely, 18 were prescribed DOACs (i.e., 2 dabigatran, 10 rivaroxaban, and 6 apixaban) and 71 warfarin. The baseline characteristics of the patients are shown in Table 1 (refer to online suppl. Table 1 for the distribution of the Japan Coma Scale; see www.karger.com/doi/10.1159/000462985 for all online suppl. material). Although the majority of baseline characteristics were comparable between the DOAC and warfarin groups, the mean hospital stay of the DOAC group (23 days; interquartile range $[\mathrm{IQR}] 13.5-34.3$ ) was significantly longer than that of the warfarin group (16 days; IQR 9.0-28.0; $p=0.04$ ). The frequency of congestive heart failure in the DOAC group was lower than that in the warfarin group, but this difference was not statistically significant. Finally, although the frequency of ICH per region was similar between the DOAC and warfarin groups, there were no cases of subcortical hemorrhage in the DOAC group. 
Adachi et al.: Cerebral hemorrhage in anticoagulation with DOACs

Table 2. Initial hematoma volume, prevalence of hematoma expansion, and mortality

\begin{tabular}{lccc}
\hline & DOAC $(n=18)$ & Warfarin $(n=71)$ & $p$ value \\
\hline Initial hematoma volume, $\mathrm{mL}$ & $6.2(2.3-18.4)$ & $24.2(5.1-48.2)$ & 0.04 \\
Prevalence of surgery & $2(11)$ & $3(4)$ & \\
Mortality & $2(11)$ & $17(24)$ & 0.34 \\
\hline
\end{tabular}

Figures are median with IQR in parentheses or numbers with percentages in parentheses. DOAC, direct oral anticoagulant; IQR, interquartile range.

\section{Initial Hematoma Size and Hematoma Expansion}

Data concerning the initial hematoma volume, follow-up hematoma volume, and frequency of hematoma expansion are shown in Table 2. A dot plot chart of hematoma volume in the DOAC and warfarin groups is shown in Figure 1. The initial hematoma volume of ICHs in the DOAC group (median: $6.2 \mathrm{~mL}$; IQR 2.3-18.4) was significantly lower than in the warfarin group (median: $24.2 \mathrm{~mL}$;QR 5.1-48.2; $p=0.04$ ). Operative therapy and early death due to a large ICH (among other reasons) did not involve follow-up CT scanning (online suppl. Table 2). A follow-up CT scan was performed in 14 cases in the DOAC group and in 53 cases in the warfarin group. The median interval between the initial and follow-up CT scans was not different between the DOAC (9.5 h; IQR 2.75-21) and warfarin (14.0 h; IQR 3.25-20) groups. The change in hematoma volume between initial and follow-up assessments was compared in the cases that could be followed up. At follow-up, although the hematoma volume was lower in the DOAC group (median: $5.0 \mathrm{~mL}$; IQR 3.6-28.0) than in the warfarin group (median: $18.4 \mathrm{~mL}$; IQR 6.5-44.5), this difference was not statistically significant ( $p=$ 0.05) (Table 3). The frequency of hematoma expansion was also not significantly different between the 2 groups (Table 3 ). However, when assessing the difference between initial and follow-up hematoma volume, the enlargement of the hematoma volume (from 13.5 to 18.4 $\mathrm{mL})$ was greater in the warfarin group $(p<0.01)$ than in the DOAC group (from 4.4 to 5.0 $\mathrm{mL}$ ) (Fig. 1).

In the warfarin group, the median international normalized ratio on admission was 2.09 (IQR 1.7-2.8). The international normalized ratio was not correlated with initial hematoma volume (online suppl. Fig. 1). In the DOAC group, we were able to identify and estimate the time from the last dose to admission in several patients. Apixaban and dabigatran were administered twice a day, while rivaroxaban was administered once a day. The number of subjects and the median time from the last dose to admission was as follows: apixaban, 4 patients, $9 \mathrm{~h}$ (IQR 3.8-11.7); dabigatran, 1 patient, $5 \mathrm{~h}$; rivaroxaban, 11 patients, $12 \mathrm{~h}$ (IQR 5.7-12). Furthermore, activated partial thromboplastin time and prothrombin time in the DOAC group was not correlated with initial hematoma volume (online suppl. Fig. 2).

In the univariate analysis, the type of anticoagulant (i.e., DOAC vs. warfarin) and the sex of the patient (i.e., male vs. female) were associated with ICH volume (Table 4). In the multivariable linear regression, age, sex, anticoagulant type, hypertension, the incidence of previous ischemic stroke, the use of DOAC (compared to warfarin; $\beta:-0.23, p=0.03$ ) and being female (compared to male; $\beta:-0.25, p=0.02$ ) were associated with a small hematoma volume.

\section{Prognosis and Mortality}

At discharge, the frequency of an mRS score of 0-3 was not significantly different between the 2 groups (i.e., $33 \%$ in DOACs vs. $17 \%$ in warfarin). Other functional prognoses at discharge were also not significantly different between the 2 groups (Fig. 2). Although the rate of 


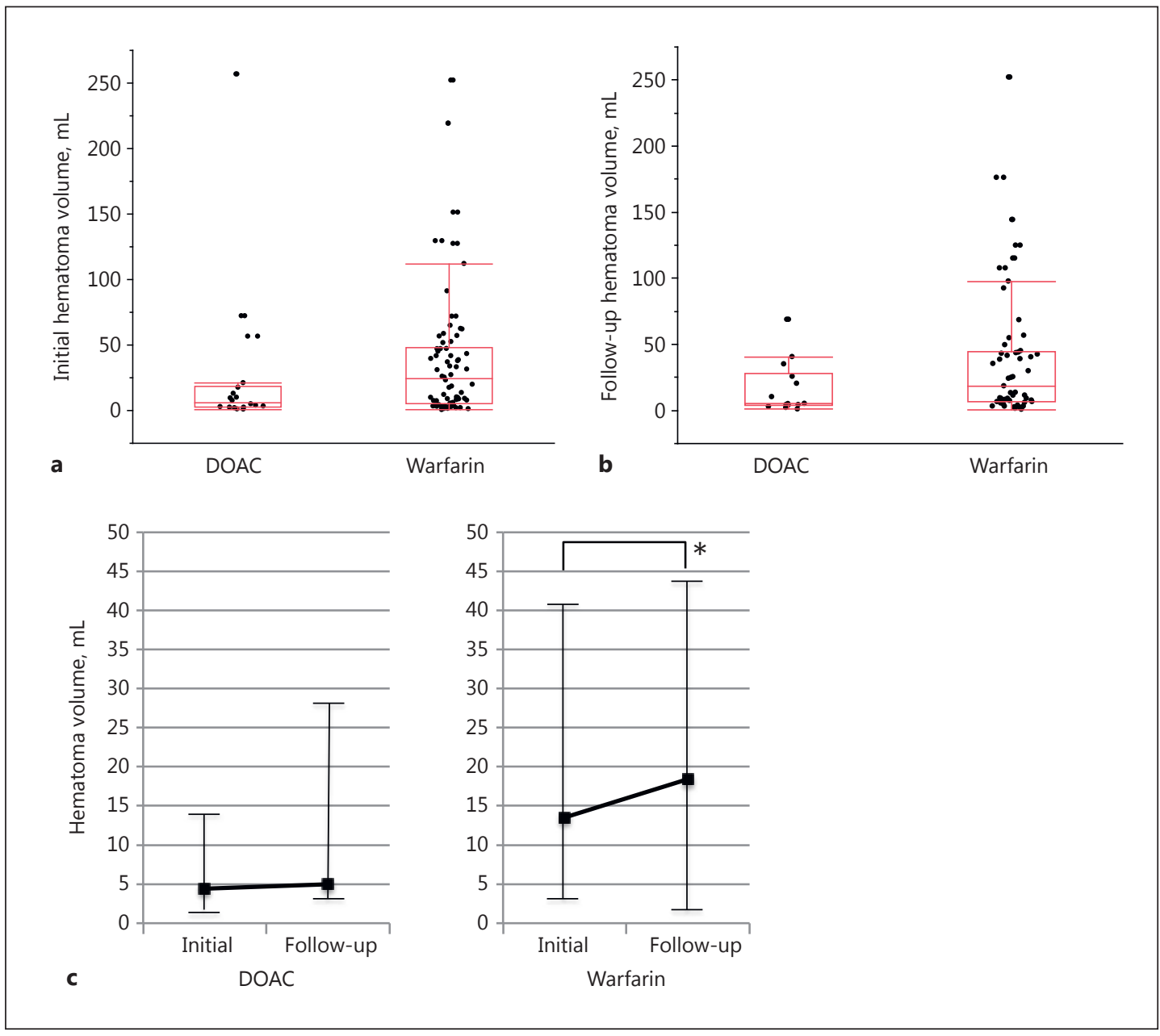

Fig. 1. Hematoma volume of ICHs associated with DOACs and warfarin. Correlation between hematoma volume and anticoagulants. Dot plots show the hematoma volume of each subject and the boxplot shows median and interquartile range of hematoma volume. a Initial hematoma volume of ICHs with DOAC and warfarin. b Follow-up hematoma volume of ICHs with DOAC and warfarin. c Change in hematoma volume between initial and follow-up. Left graph shows ICHs with DOAC, and right graph shows ICHs with warfarin. Graph shows median and interquartile range of hematoma volume. ${ }^{*} p<0.01$. ICH, intracranial hemorrhage; DOAC, direct oral anticoagulants.

Table 3. Initial hematoma volume, follow-up hematoma volume, and prevalence of hematoma expansion in follow-up cases

\begin{tabular}{lccc}
\hline & DOAC $(n=14)$ & Warfarin $(n=53)$ & $p$ value \\
\hline Initial hematoma volume, mL & $4.4(2.3-14.1)$ & $13.5(3.7-41.6)$ & 0.04 \\
Follow-up hematoma volume, mL & $5.0(3.6-27.9)$ & $18.4(2.7-44.5)$ & 0.05 \\
Prevalence of hematoma expansion & $9(64)$ & $27(51)$ & 0.54 \\
Initial to follow-up time, h & $9.5(2.75-21)$ & $14(3.25-20)$ & 0.48 \\
Mortality & $2(11)$ & $17(24)$ & 0.34 \\
\hline
\end{tabular}

Figures are medians with IQRs in parentheses or numbers with percentages in parentheses. DOAC, direct oral anticoagulant; IQR, interquartile range. 
Table 4. Univariate analysis of predictors associated with mean ICH volume

\begin{tabular}{llll}
\hline Predictor & Mean \pm SD & $95 \%$ CI & $p$ value \\
\hline Age, $<75$ vs. $\geq 75$ years & $2.85 \pm 1.50$ vs. $2.52 \pm 1.48$ & $2.30-3.39$ vs. $2.12-2.92$ & 0.33 \\
Anticoagulant type, DOAC vs. warfarin & $2.00 \pm 1.54$ vs. $2.80 \pm 1.44$ & $1.02-2.61$ vs. $2.21-3.11$ & 0.040 \\
Sex, female vs. male & $2.20 \pm 1.52$ vs. $2.97 \pm 1.39$ & $1.24-2.51$ vs. $2.03-2.98$ & 0.015 \\
Hypertension, yes vs. no & $2.62 \pm 1.51$ vs. $2.69 \pm 1.44$ & $1.93-2.77$ vs. $1.29-2.97$ & 0.88 \\
Previous ischemic stroke, yes vs. no & $2.68 \pm 1.37$ vs. $2.56 \pm 1.76$ & $1.78-2.83$ vs. $1.48-2.86$ & 0.73 \\
\hline
\end{tabular}

ICH, intracerebral hemorrhage; DOAC, direct oral anticoagulant; SD, standard deviation; CI, confidence interval.

Fig. 2. Proportion of modified Rankin Scale (mRS) score at discharge. mRS at discharge compared between ICHs associated with DOAC and warfarin. Each number is $n(\%)$. ICH, intracranial hemorrhage; DOAC, direct oral anticoagulants.

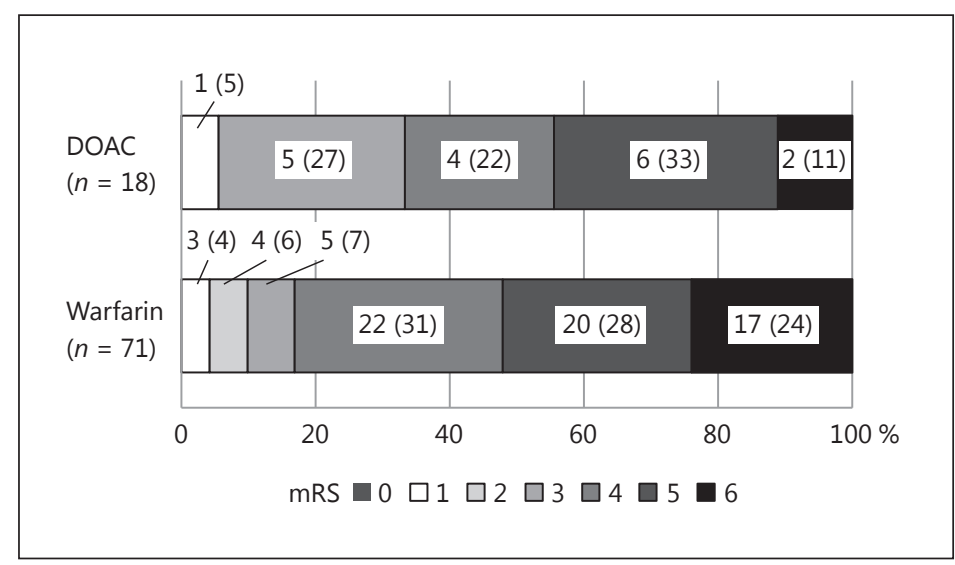

mortality, due to any cause, at discharge was higher in the warfarin group (17 cases; $24 \%$ ) than in the DOAC group (2 cases; 11\%), this difference was not statistically significant (Table 2).

\section{Discussion}

In this prospective multicenter cross-sectional study, we showed that the initial hematoma volume in patients treated with DOACs was significantly lower than in those treated with warfarin. The follow-up hematoma volume and the mortality rates were also lower in the DOAC group than in the warfarin group (although these differences were not statistically significant).

Previous randomized controlled trials of DOACs showed that the incidence of ICHs with the use of DOACs was significantly lower than with warfarin [7-10]. We hypothesized that the incidence of ICHs would be lower following therapy with DOACs and that the hematoma volume associated with DOACs would be lower compared with warfarin. Our results supported these hypotheses. In some previous Japanese studies, it was reported that ICHs associated with DOAC were small and had good clinical outcomes compared to those associated with warfarin [14, 15]. Recently, Wilson et al. [18] reported that, compared with warfarin, the hematoma volume of ICHs associated with DOACs was significantly lower, with better functional outcomes. Importantly, the authors also suggested that these results could be generalized to other populations [18]. The findings of the current study corroborated these findings. 
The results of the current study and several previous studies $[14,15,18]$ also suggest that ICHs associated with DOACs are smaller than those associated with warfarin, which is true in different populations, including the Asian population, a high-risk population for ICHs associated with anticoagulant therapy.

In the multivariate analysis, the use of DOACs and being female were associated with a small hematoma volume. In females, the hematoma volume of ICHs was not different between the DOAC group and warfarin group. Conversely, in males, the hematoma volume of ICHs in the DOAC group was significantly lower in comparison with the warfarin group (data shown in online suppl. Fig. 3). The reasons for this result are unknown and require further study.

We also demonstrated that at follow-up, the hematoma volume of ICHs associated with DOAC was lower than that associated with warfarin, and that enlargement of the hematoma volume was much greater in the warfarin group. This is a novel finding, which we postulate is related to the different mechanism of action of DOACs and warfarin [18-21].

In the current study, the mortality rate associated with DOAC-induced ICHs at discharge was lower than that associated with warfarin-induced ICHs. In the original clinical trials on DOACs (RE-LY [7], ARISTOTLE [9], ROCKET AF [8], and ENGAGE AF TIMI 48 [10]), although the incidence of ICHs associated with DOAC was significantly lower than the incidence of ICHs associated with warfarin, there was no difference in the mortality rate [7-10]. The reasons for this were not explored. The results of the present study are important because they demonstrate the safety of DOACs in real-world clinical situations. However, given the small sample, we could not demonstrate a statistically significant difference in terms of mortality rate. Thus, we believe that future studies with a larger patient cohort will help establish if there are mortality differences due to ICHs associated with these 2 treatments.

There are some limitations in the present study. First, the follow-up hematoma volume was not measured in all subjects, as we could not perform follow-up brain CT scans for patients who underwent emergency surgery or experienced death due to a massive ICH. We believe that it was acceptable not to perform follow-up CT scans for these patients since there was no clinical need to reevaluate hematoma volume. Second, the evaluation of mortality and prognoses was performed at discharge rather than at a fixed time point. Previous studies revealed that death related to ICHs usually occurred within the first week from onset [22]. The median length of hospital stay was 23 days in the DOAC group and 16 days in the warfarin group. Therefore, we thought that mortality at discharge sufficiently estimated the early prognosis of ICHs. Finally, acute-phase management of ICHs was not consistent among the institutes included in the study. However, since the management of ICHs tends to vary among institutes in actual clinical practice, we considered it an advantage, as the results of this observational study was not based on an idealized treatment scenario. We believe that the results of the present study are closer to real-world clinical practice than those of previously published reports. However, further studies are required to validate the results of the present study. Future research should also evaluate individual DOACs in order to determine if these drugs are equivalent in terms of the risks associated with ICHs.

In conclusion, the initial hematoma volume in patients treated with DOACs was significantly lower than that in patients treated with warfarin, which was true at follow-up. DOACs appear to offer advantages in terms of better safety over warfarin for anticoagulation therapy. Further studies involving a larger patient sample size are required to confirm these findings.

\section{Acknowledgements}

We gratefully thank the members of the Saiseikai Stroke Research Group for clinical data collection and advice on this study. 

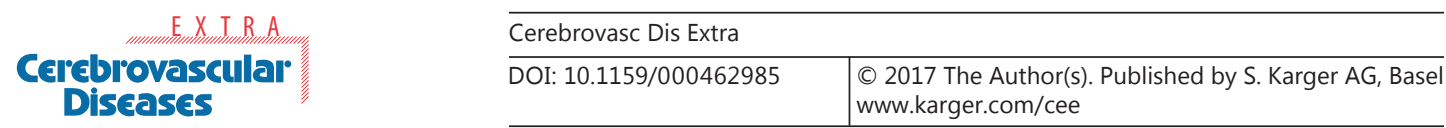

Adachi et al.: Cerebral hemorrhage in anticoagulation with DOACs

\section{Disclosure Statement}

Tomohide Adachi, MD has received honoraria for speaking at an event organized by the Nippon Boehringer-Ingelheim Co., Ltd. Haruhiko Hoshino, MD has received honoraria and travel expenses for speaking at an event organized by the Sanofi K.K., Otsuka Pharmaceutical Co., Ltd., Nippon Boehringer-Ingelheim Co., Ltd., Bayer Yakuhin Ltd., and Bristol-Myer Squibb Company Co., Ltd. Makoto Takagi, MD has received honoraria for speaking at an event organized by Pfizer Co., Ltd., Daiichi-Sankyo Co., Ltd., and Nippon Boehringer-Ingelheim Co., Ltd. Shodo Fujioka, MD has nothing to disclosure.

\section{Funding Sources}

This study was supported by grants for medical and welfare collaborate investigation (Construction of Saiseikai Stroke Database) from the Social Welfare Organization Saiseikai Imperial Gift Foundation.

\section{Appendix}

\section{Saiseikai Stroke Research Group}

Shuichi Mori: Mito Saiseikai General Hospital, neurosurgery; Kentaro Furusho: Ryugasaki Saiseikai Hospital, neurology; Sumio Ishimaru: Saiseikai Kawaguchi General Hospital, neurosurgery; Hiroshi Wanifuchi: Saiseikai Kurihashi Hospital, neurosurgery; Makoto Takagi, Haruhiko Hoshino, Tomohide Adachi: Tokyo Saiseikai Central Hospital, neurology; Michiyuki Maruyama: Saiseikai Yokohamashi Tobu Hospital, strokology and neurology; Hitoshi Ohishi: Saisekai Yokohamashi Nanbu Hospital, neurosurgery; Tsuneo Nakamura: Saiseikai Wakakusa Hospital, neurosurgery; Yukio Horie, Michiya Kubo: Saiseikai Toyama Hospital, neurosurgery; Eiichi Uno, Yasushi Takabatake: Fukui-ken Saiseikai Hospital, neurosurgery; Masahide Kondou, Toshiyuki Sakai: Saiseikai Matsuzaka General Hospital, neurology; Akihiko Hino; Saiseikai Shiga Hospital, neurosurgery; Hedeki Oka, Akihiro Fujii: Saiseikai Shiga Hospital, neurology; Nobukuni Murakami: Saiseikai Kyoto Hospital, neurosurgery; Katsuhiko Kono: Saiseikai Noe Hospital, neurosurgery; Toyohisa Fujita: Saiseikai Tyuwa Hospital, neurosurgery; Yutaka Naka: Saiseikai Wakayama Hospital, neurosurgery; Yasuaki Aoyama: Saiseikai Sakaiminato General Hospital, neurology; Yukio Kato: Saiseikai Hiroshima Hospital, neurosurgery; Tetsuhiro Kitahara: Saiseikai Yamaguchi General Hospital, neurosurgery; Katsusuke Kusunoki: Saiseikai Matsuyama Hospital, neurosurgery; Hiroshi Uramoto, Katsusuke Kusunoki: Saiseikai Saijo Hospital; Takeshi Yamada: Saiseikai Fukuoka General Hospital, neurology; Yuuji Okamoto: Saiseikai Yahata General Hospital, neurosurgery; Toru Nishi, Takamasa Mizuno: Saiseikai Kumamoto Hospital, neurosurgery; Shodo Fujioka: Saiseikai Misumi Hospital, neurosurgery.

\section{References}

1 Flaherty ML, Tao H, Haverbusch M, Sekar P, Kleindorfer D, Kissela B, et al: Warfarin use leads to larger intracerebral hematomas. Neurology 2008;71:1084-1089.

-2 Flibotte JJ, Hagan N, O'Donnell J, Greenberg SM, Rosand J: Warfarin, hematoma expansion, and outcome of intracerebral hemorrhage. Neurology 2004;63:1059-1064.

-3 Toyoda K, Yasaka M, Iwade K, Nagata K, Koretsune Y, Sakamoto T, et al: Dual antithrombotic therapy increases severe bleeding events in patients with stroke and cardiovascular disease: a prospective, multicenter, observational study. Stroke 2008;39:1740-1745. 
4 Kuwashiro T, Yasaka M, Itabashi R, Nakagaki H, Miyashita F, Naritomi H, et al: Enlargement of acute intracerebral hematomas in patients on long-term warfarin treatment. Cerebrovasc Dis 2010;29:446-453.

5 Hankey GJ, Stevens SR, Piccini JP, Lokhnygina Y, Mahaffey KW, Halperin JL, et al: Intracranial hemorrhage among patients with atrial fibrillation anticoagulated with warfarin or rivaroxaban: the rivaroxaban once daily, oral, direct factor Xa inhibition compared with vitamin $\mathrm{K}$ antagonism for prevention of stroke and embolism trial in atrial fibrillation. Stroke 2014;45:1304-1312.

6 Shen AY, Yao JF, Brar SS, Jorgensen MB, Chen W: Racial/ethnic differences in the risk of intracranial hemorrhage among patients with atrial fibrillation. J Am Coll Cardiol 2007;50:309-315.

7 Connolly SJ, Ezekowitz MD, Yusuf S, Eikelboom J, Oldgren J, Parekh A, et al: Dabigatran versus warfarin in patients with atrial fibrillation. N Engl J Med 2009;361:1139-1151.

8 Patel MR, Mahaffey KW, Garg J, Pan G, Singer DE, Hacke W, et al: Rivaroxaban versus warfarin in nonvalvular atrial fibrillation. N Engl J Med 2011;365:883-891.

-9 Granger CB, Alexander JH, McMurray JJ, Lopes RD, Hylek EM, Hanna M, et al: Apixaban versus warfarin in patients with atrial fibrillation. N Engl J Med 2011;365:981-992.

10 Giugliano RP, Ruff CT, Braunwald E, Murphy SA, Wiviott SD, Halperin JL, et al: Edoxaban versus warfarin in patients with atrial fibrillation. N Engl J Med 2013;369:2093-2104.

11 Hori M, Connolly SJ, Zhu J, Liu LS, Lau CP, Pais P, et al: Dabigatran versus warfarin: effects on ischemic and hemorrhagic strokes and bleeding in Asians and non-Asians with atrial fibrillation. Stroke 2013;44:18911896.

12 Wong KS, Hu DY, Oomman A, Tan RS, Patel MR, Singer DE, et al: Rivaroxaban for stroke prevention in east Asian patients from the ROCKET AF trial. Stroke 2014;45:1739-1747.

13 Goto S, Zhu J, Liu L, Oh BH, Wojdyla DM, Aylward P, et al: Efficacy and safety of apixaban compared with warfarin for stroke prevention in patients with atrial fibrillation from East Asia: a subanalysis of the Apixaban for Reduction in Stroke and Other Thromboembolic Events in Atrial Fibrillation (ARISTOTLE) Trial. Am Heart J 2014;168:303-309.

14 Komori M, Yasaka M, Kokuba K, Matsuoka H, Fujimoto S, Yoshida M, et al: Intracranial hemorrhage during dabigatran treatment - case series of eight patients. Circ J 2014;78:1335-1341.

15 Saji N, Kimura K, Aoki J, Uemura J, Sakamoto Y: Intracranial hemorrhage caused by non-vitamin K antagonist oral anticoagulants (NOACS) - multicenter retrospective cohort study in Japan. Circ J 2015;79:1018-1023.

16 Japanese Guidelines for the Management of Stroke 2009. Appendix. J Stroke Cerebrovasc Dis 2011;20:S181S196.

17 Kothari RU, Brott T, Broderick JP, Barsan WG, Sauerbeck LR, Zuccarello M, et al: The ABCs of measuring intracerebral hemorrhage volumes. Stroke 1996;27:1304-1305.

18 Wilson D, Charidimou A, Shakeshaft C, Ambler G, White M, Cohen H, et al: Volume and functional outcome of intracerebral hemorrhage according to oral anticoagulant type. Neurology 2016;86:360-366.

19 Colman RW: Are hemostasis and thrombosis two sides of the same coin? J Exp Med 2006;203:493-495.

20 Fleck RA, Rao LV, Rapaport SI, Varki N: Localization of human tissue factor antigen by immunostaining with monospecific, polyclonal anti-human tissue factor antibody. Thromb Res 1990;59:421-437.

21 Lauer A, Cianchetti FA, Van Cott EM, Schlunk F, Schulz E, Pfeilschifter W, et al: Anticoagulation with the oral direct thrombin inhibitor dabigatran does not enlarge hematoma volume in experimental intracerebral hemorrhage. Circulation 2011;124:1654-1662.

22 Bamford J, Dennis M, Sandercock P, Burn J, Warlow C: The frequency, causes and timing of death within 30 days of a first stroke: the Oxfordshire Community Stroke Project. J Neurol Neurosurg Psychiatry 1990;53: 824-829. 